\title{
The Relationship Between Circulating Bone Morphogenetic Protein-4 and Inflammation Cytokines in Patients Undergoing Thoracic Surgery: A Prospective Randomized Study
}

\author{
Xu Zhaol,* \\ Jitao Zhang ${ }^{1} *$ \\ Wenjuan Zhang ${ }^{2}$ \\ Ruping Dai ${ }^{1}$ \\ Junmei $X u^{\prime}$ \\ Zhijian Li' \\ Lin Yang $\mathbb{D}^{\prime}$

\begin{abstract}
'Department of Anesthesiology, The Second Xiangya Hospital, Central South University, Changsha, Hunan, 4I00II, People's Republic of China; ${ }^{2}$ Department of Laboratory Medicine, The Second Xiangya Hospital, Central South University, Changsha, Hunan, 4I00II, People's Republic of China

*These authors contributed equally to this work
\end{abstract}

Background: Bone morphogenetic protein-4 (BMP4) has been identified as an inflammation regulator in the diseases of arteries and other organs. However, the relationship between circulating BMP4 and perioperative inflammation remains unclear.

Patients and Methods: Forty patients undergoing lobectomy were randomly allocated into the Control group (not receiving flurbiprofen) and the Flurb group (received 100mg flurbiprofen during surgery). Arterial blood was obtained before surgery (T1), at the end of surgery (T2), and 24 hours after surgery (T3) to test the plasma concentrations of BMP4, its antagonist Noggin, interleukin (IL)-1 $\beta$, tumor necrosis factor- $\alpha$ (TNF- $\alpha$ ), and IL-10. The relationship between BMP4 and other variables and the effects of flurbiprofen on BMP4 changes were investigated.

Results: A total of 35 patients were included. Circulating BMP4 was positively correlated with IL-1 $\beta(P<0.01, \mathrm{r}=0.575)$ and TNF- $\alpha(P<0.01, \mathrm{r}=0.491)$, negatively correlated with IL-10 $(P<0.01, \mathrm{r}=-0.675)$, but not correlated with Noggin. The plasma concentrations of BMP4, IL-1 $\beta$, and TNF- $\alpha$ increased at T2 $(P<0.01$, compared with T1) and decreased at T3 $(P<0.05$, compared with T2). BMP4 concentrations at T3 were significantly higher than at T1 in the Control group $(P<0.05)$, while showing no significant difference in the Flurb group. However, in the Flurb group, the relative changes of BMP4 and IL-1 $\beta$ at T2 and T3 were significantly lower than those in the Control group.

Conclusion: Circulating BMP4 was elevated during surgery and highly correlated with inflammation cytokines. The elevation of BMP4 and inflammatory cytokines could be alleviated by flurbiprofen, indicating that BMP4 may exert pro-inflammatory properties via cyclooxygenase-II signaling pathways.

Keywords: bone morphogenetic protein-4, Noggin, inflammation, non-steroidal antiinflammatory drugs, perioperative, cyclooxygenase-II

\section{Introduction}

Surgical trauma and other invasive interventions (eg, mechanical ventilation, punctures, and blood transfusion) are unavoidable to trigger inflammation perioperatively. ${ }^{1}$ Current thinking suggests that inflammation can exert both beneficial and detrimental effects on the body, depending on the extent and duration of the inflammatory phase. ${ }^{2,3}$ Notably, when over-activated, inflammation can cause a series of severe clinical consequences, including sepsis, multiple organ
Correspondence: Lin Yang; Zhijian Li Department of Anesthesiology, The Second Xiangya Hospital, Central South University, Changsha, Hunan, 4I00II,

People's Republic of China

Fax +86 73185295970 ;

Fax +8673185295970

Email linyang@csu.edu.cn;

lizhijian@csu.edu.cn 
dysfunction syndrome (MODS), and cancer recurrence, all of which can prove grave for patients. ${ }^{4}$ Thus, clarification of the clinical regulatory mechanism of inflammation is urgently required.

Bone morphogenetic protein-4 (BMP4) is a member of the transforming growth factor $\beta$ (TGF- $\beta$ ) superfamily. Previous studies have demonstrated the critical role that BMP4 plays in dictating tissue homeostasis and disease in adults postinjury. ${ }^{5-7}$ Crucially, BMP4 can be synthesized, stored, and released by the vascular endothelial cell, ${ }^{8}$ cardiomyocyte, ${ }^{9}$ and adipocyte. ${ }^{10}$ Recent experimental data shows that BMP4 expressed via the systematic arterial endothelium could induce endothelial dysfunction, promote leukocytes activation, and aggravate the severity of atherosclerosis. ${ }^{11}$ BMP4 expressed by cardiomyocytes following myocardial infarction could increase infarct size through upregulating pro-inflammatory factors, including interleukin (IL)-1 $\beta$ and IL-9. ${ }^{12,13}$ Moreover, corroborating evidence ${ }^{11,14}$ further proves that BMP4 signaling is mainly linked to the induction of inflammatory nuclear factor- $\mathrm{\kappa B}(\mathrm{NF}-\mathrm{\kappa B})$, nicotinamide adenine dinucleotide phosphate oxidase-1 (NOX1), and intracellular adhesions molecule-1 (ICAM-1), which are the key factors at the initial inflammation stage. All this evidence indicates that BMP4 elevation will exert pro-inflammatory properties at the lesion site of systemic arteries and the heart. In humans, however, the clinical relationship between circulating BMP4 and inflammation still remains unclear.

In the present study, we planned to enroll patients undergoing lobectomy and investigate their plasma concentrations of BMP4, its antagonist, and a series of canonical pro- and anti-inflammatory cytokines, including IL-1 $\beta$, tumor necrosis factor- $\alpha$ (TNF- $\alpha$ ), and IL-10. Furthermore, we tested the effects of flurbiprofen - a non-steroidal anti-inflammatory drug - on the changes of the above variables. We chose Noggin as the antagonist for BMP4 because it has a high affinity to de-activate the BMP4 signaling ${ }^{15}$ and has been validated in previous studies. ${ }^{16,17}$ The results may help to heighten our understanding of the relationship between circulating BMP4 and inflammation, as well as assist in the identification of a new candidate for treating perioperative inflammation in the future.

\section{Methods}

The Second Xiangya Hospital's Institutional Review Board (IRB \#LYG2021011) approved this study, and written informed consent was obtained from all subjects participating in the trial. The trial was registered prior to patient enrollment at the Chinese Clinical Trial Registry (www.chictr.org.cn; ChiCTR2000038374; principal investigator: Lin Yang; date of registration: September 21st, 2020) and conducted in accordance with the principles of the Declaration of Helsinki.

\section{Patients}

Patients with an American Society of Anesthesiologists physical status of I or II undergoing lobectomy through thoracoscopic technique at the Second Xiangya Hospital, Central South University, were screened for study eligibility. Considering that circulating BMP4 may increase in diseases that include atherosclerosis, ${ }^{11}$ hypertension, ${ }^{11}$ myocardial infarction, ${ }^{12}$ diabetes, ${ }^{18,19}$ and obesity, ${ }^{20}$ the following patients were excluded: 1) patients with vascular diseases especially vascular sclerosis or diagnosed plaque formation; 2) patients with cardiac, hepatic, and other vital organ diseases; 3) patients with hypertension and diabetes; 4) patients with a body mass index (BMI) above $30 \mathrm{~kg} / \mathrm{m}^{2} ; 5$ ) patients with a history of drug abuse, receiving long-term steroid therapy, or allergic to non-steroidal antiinflammatory drugs; 6) patients with an increased (above the upper normal limit) white blood cell count, neutrophils ratio, erythrocyte sedimentation rate, or procalcitonin before surgery; and 7) when the type of surgery changed, or the blood loss exceeded $1000 \mathrm{~mL}$ during surgery.

\section{Randomization and Allocation}

Patients were randomly assigned to either the Control or Flurb group in a 1:1 ratio, with the random list generated using the Microsoft Excel' RAND' function. After randomization, a researcher who did not participate in the anesthesia management prepared 'study drugs' containing either lipid emulsion $(10 \mathrm{~mL})$ or flurbiprofen (100mg, $10 \mathrm{~mL}$ ) outside the operating room. The anesthesiologists in charge of the whole anesthesia management were kept blind to the group allocation.

\section{Anesthesia and Intervention}

Routinely, after entering the operation room, each patient was monitored with an electrocardiograph, invasive arterial pressure, pulse oximetry, and Bispectral index (BIS). General anesthesia was induced by intravenous injections of midazolam $0.05 \mathrm{mg} / \mathrm{kg}$, etomidate $0.1 \mathrm{mg} / \mathrm{kg}$, sufentanil $0.5 \mu \mathrm{g} / \mathrm{kg}$, and cis-atracurium $0.1 \mathrm{mg} / \mathrm{kg}$. After the patients' eyelid reflections disappeared and muscle relaxation was achieved, the same anesthesiologist performed doublelumen tracheal (DLT) intubation under the vision of a video laryngoscope and further confirmed the depth of 
DLT by a bronchoscope. Anesthesia, analgesia, and muscle relaxation were maintained by continuous infusions of propofol $6-8 \mathrm{mg} / \mathrm{kg} / \mathrm{h}$, remifentanil $0.3 \mu \mathrm{g} / \mathrm{kg} / \mathrm{min}$, and intermittent injections of cis-atracurium. During the operation, end-expiratory carbon dioxide was adjusted between $35-45 \mathrm{mmHg}$, and anesthesia depth was maintained between $40-50$ of BIS values. The infusion of all anesthetics halted upon skin closure. Subsequently, $0.1 \mu \mathrm{g} / \mathrm{kg}$ sufentanil was injected and a patient-controlled analgesia pump containing sufentanil $150 \mu \mathrm{g}$ and ondansetron $16 \mathrm{mg}$ in $100 \mathrm{~mL}$ normal saline was connected intravenously to each patient to treat post-operative pain. All patients were transferred to a thoracic surgical intensive care unit postoperatively.

In the Flurb group, $100 \mathrm{mg}$ flurbiprofen was infused intravenously at the time of skin incision, within $30 \mathrm{~min}$ utes, while patients in the Control group were treated with an equal volume of lipid emulsion at the same time.

\section{Data Collections}

Arterial blood samples were drawn from each patient at three time points: T1 (before the surgery), T2 (the end of the surgery), and T3 (24 hours after the surgery). The blood samples were stored in tubes containing ethylene diamine tetraacetate acid and were further processed within 12 hours. Each sample was centrifuged for $15 \mathrm{~min}$ at $3000 \mathrm{rpm}$ to obtain the plasma. Then, the levels of BMP4 (ab231930, Abcam, Cambridge, UK), Noggin (JYM2457Hu, Wuhan, CHN), IL-1 $\beta$ (ab217608, Abcam, Cambridge, UK), TNF- $\alpha$ (ab181421, Abcam, Cambridge, UK), and IL-10 (ab185986, Abcam, Cambridge, UK) were determined using the enzyme-linked immunosorbent assays method (SimpleStep ELISA $^{\circledR}$ kits, Abcam, Cambridge, UK) by a researcher who was blind to the group allocation.

\section{Statistical Analysis}

For the sample size calculation, we assumed there to be a correlation (|correlation coefficient $\mid>0.3$ ) between BMP4 and other inflammatory cytokines. We chose a correlation coefficient of 0.3 because it was the smallest value representing a non-negligible correlation, as suggested by a previous study. ${ }^{21}$ Using a smaller size of correlation in sample size calculation would require more patients, but it was a conservative way to avoid underpower. With a twosided significance level of 0.05 and a power of $80 \%, 85$ samples were required. Assuming a drop-out rate of approximately $25 \%$, we planned to enroll 40 patients, with a sample size of 120 ( 40 patients*3 time points).

Continuous data were presented as median and interquartile range (IQR), while categorical data were presented as frequency and percentage. Two analyses were performed to investigate the relationship between BMP4 and inflammation (Figure 1). Firstly, we conducted pairwise comparisons among BMP4, Noggin, and inflammatory cytokines using Spearman's rank correlation coefficient ( $\mathrm{r}$ ) based on samples from both groups. A $|\mathrm{r}|$ $>0.7$ was identified as having a strong correlation; $a|r|=$ $0.3-0.7$ was identified as having low to moderate correlation; and a $|r|<0.3$ was identified as having no correlation. $^{21}$ Scatter plots and fitted linear regression lines were used to visualize the relationship between two variables. Secondly, we evaluated the effects of flurbiprofen on BMP4, Noggin, and other inflammatory cytokines. Their plasma concentrations at different time points were analyzed using the Kruskal-Wallis test, followed by Dunn's multiple comparison test. Because the absolute values of BMP4, Noggin, and other inflammatory cytokines varied considerably, we further calculated the relative changes of variables to investigate the effects of

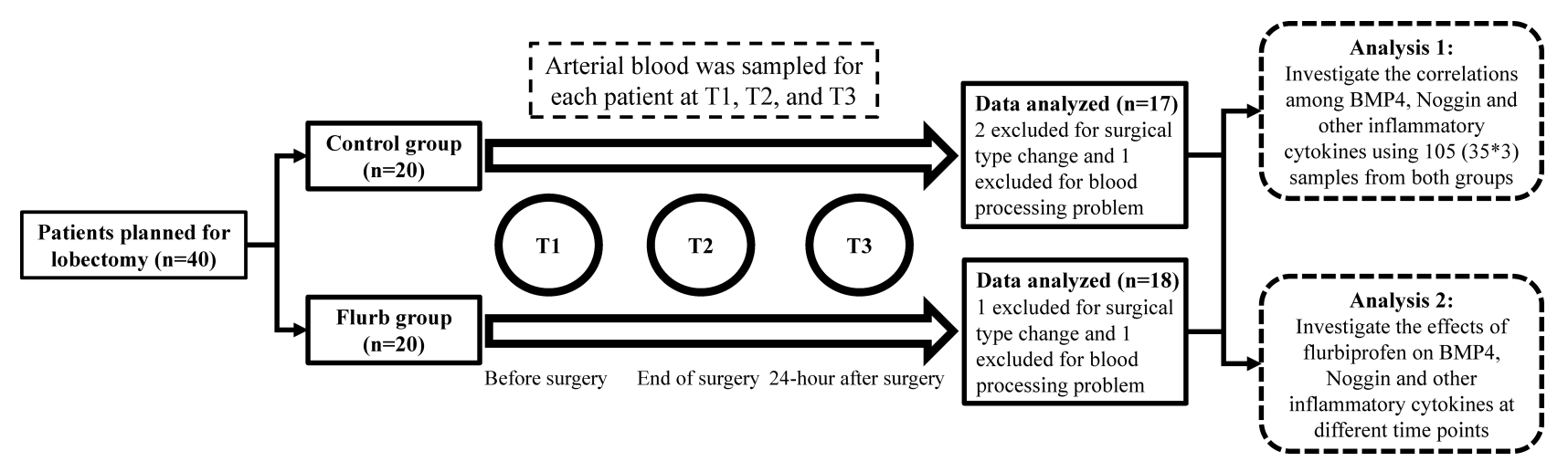

Figure I Flow chart.

Abbreviations: Flurb, flurbiprofen; BMP4, bone morphogenetic protein-4. 
flurbiprofen. The relative changes indicated the ratio of the concentrations at different time points versus the concentration at T1. The relative changes in Control and Flurb groups were compared using the Mann-Whitney $U$-test.

All analyses were performed using SPSS software (version 19.0, IBM, NY, US), Prism software (version 8.0.1, GraphPad Inc., CA, USA), and R software (version 3.5.3, $\mathrm{R}$ Foundation for Statistical Computing, Vienna, Austria). A $P<0.05$ was considered to be statistically significant.

\section{Results}

From October 1st, 2020, to January 31st, 2021, we enrolled 40 patients. From this batch, five patients were excluded for the following reasons: three due to the change of surgery, in which the surgical resection extended and the operation duration was prolonged; and two due to the technical problems of blood processing. Overall, 35 patients with a total of 105 samples were included in the analyses, for which the flow chart is shown in Figure 1. The general information (including gender, age, and BMI) and surgical characteristics (including duration of surgery, blood loss, and fluid therapy) of patients from each group are presented in Table 1.

The correlations among plasma concentrations of BMP4, Noggin, and different inflammatory cytokines are shown in Figure 2. BMP4 was positively correlated with IL-1 $\beta(\mathrm{r}=0.575, P<0.01)$ and TNF- $\alpha(\mathrm{r}=0.491, P<0.01)$, but negatively correlated with IL-10 ( $\mathrm{r}=-0.675, P<0.01)$. In contrast, Noggin did not show any correlation with either BMP4 $\quad(\mathrm{r}=-0.034, \quad P=0.73), \quad \mathrm{IL}-1 \beta \quad(\mathrm{r}=0.030$, $P=0.76)$, TNF $-\alpha(\mathrm{r}=-0.055, P=0.58)$, or $\mathrm{IL}-10(\mathrm{r}=0.106$, $P=0.28)$.

The plasma concentrations for all variables at different time points are presented in Table 2 and Figure 3. Overall, the concentrations of BMP4, IL- $1 \beta$, TNF- $\alpha$, and IL-10

Table I Patients' General Information and Surgical Characteristics

\begin{tabular}{|l|c|c|}
\hline & Control Group (n=17) & $\begin{array}{c}\text { Flurb Group } \\
(\mathbf{n}=18)\end{array}$ \\
\hline Gender, male & $10(58.8 \%)$ & $12(66.7 \%)$ \\
Age, year & $53(48-57)$ & $54(49-58)$ \\
BMl, kg/m² & $22.3(21.5-23.7)$ & $22.5(21.5-24.1)$ \\
Duration of surgery, h & $2.5(2.0-3.5)$ & $3.0(2.5-3.5)$ \\
Blood loss, $\mathrm{mL}$ & $50(50-50)$ & $50(50-68)$ \\
Fluid therapy, $\mathrm{mL}$ & $1000(700-1000)$ & $1000(800-1000)$ \\
\hline
\end{tabular}

Note: Data presented as frequency (percentage) or median (interquartile range). Abbreviations: Flurb, flurbiprofen; BMI, body mass index. fluctuated (Kruskal-Wallis $P<0.01$, Table 2), while the Noggin concentrations stayed relatively stable at different time points (Kruskal-Wallis $P=0.40$ and 0.87 , respectively for each group, Table 2). Specifically, in both groups, the plasma concentrations of BMP4, IL-1 $\beta$, and TNF- $\alpha$ increased at T2 $(P<0.01$, compared with T1, Figure 3$)$ and decreased at T3 $(P<0.05$, compared with T2, Figure 3), while IL-10 levels were downregulated at T2 $(P<0.01$, compared with T1, Figure 3$)$ and elevated at T3 $(P<0.01$, compared with $\mathrm{T} 2$, Figure 3$)$. Then, in the Control group, concentrations of BMP4, IL-1 $\beta$, and TNF- $\alpha$ at T3 were markedly higher than at T1 $(P<0.05$, Figure 3), and IL-10 levels at T3 were significantly lower than at $\mathrm{T} 1 \quad(P<0.05$, Figure 3$)$. In contrast, in the Flurb group, concentrations of BMP4, IL- $1 \beta$, TNF- $\alpha$, and IL-10 at $\mathrm{T} 1$ and $\mathrm{T} 3$ did not show a significant difference (Figure 3). Finally, the Noggin concentration did not show any differences among three time points (Figure 3 ).

The relative changes of BMP4, Noggin, IL- $1 \beta$, TNF- $\alpha$, and IL-10 at different time points were listed in Table 3. In the Flurb group, the relative changes of BMP4 and IL-1 $\beta$ were significantly lower than those in the Control group at T2 and T3 $(P<0.05)$. Otherwise, no significant differences were found between the two groups.

\section{Discussion}

This is the first human study investigating the relationship between circulating BMP4 and inflammation in perioperative settings. The results show some novel findings that have never been reported: 1) circulating BMP4 is positively correlated with pro-inflammatory cytokines (IL-1 $\beta$ and TNF- $\alpha$ ), but negatively correlated with antiinflammatory factor (IL-10), indicating that BMP4 is highly associated with inflammatory responses; 2) BMP4 increased at the end of surgery and decreased 24-hours after surgery, indicating that BMP4 could serve as a new inflammatory indicator during surgery; 3) the application of $100 \mathrm{mg}$ flurbiprofen during surgery decreased BMP4 and IL-1 $\beta$ levels, indicating that circulating BMP4 might regulate inflammation via the cyclooxygenase-II (COX-2) pathways.

BMP4 has now been identified as a novel inflammation regulator in multiple organs. Studies from the last two decades have shown conflicting results on whether BMP4 could act as a molecular switch that induces both pro-inflammatory and anti-inflammatory effects on special organs. Except for BMP4's pro-inflammatory properties in systemic arteries and myocardium, ${ }^{11-13}$ the elevation of 

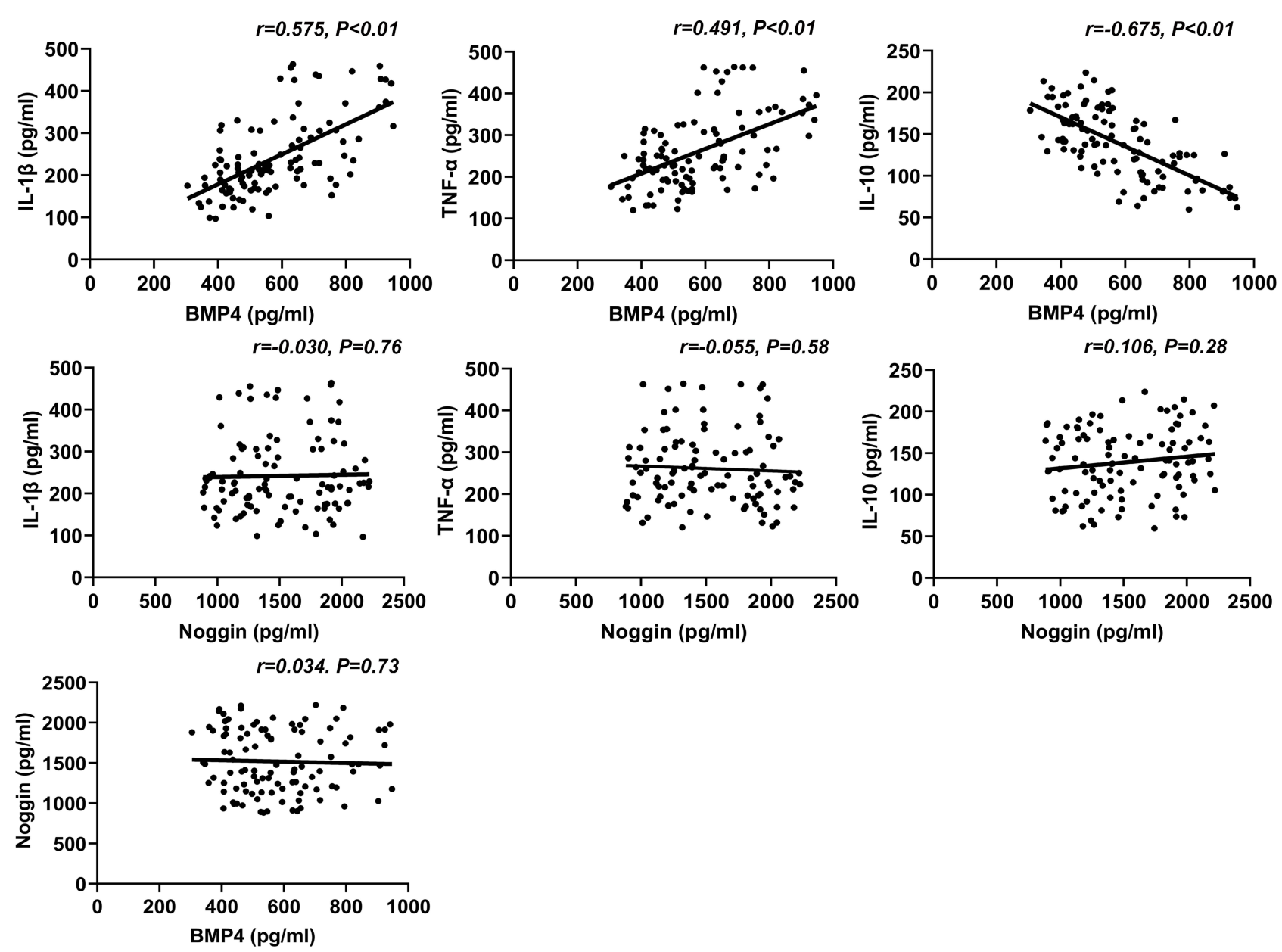

Figure 2 Correlations among plasma concentrations of BMP4, Noggin, and inflammatory cytokines. The Spearman's rank correlation coefficient ( $r$ ) and the correlation test's significance level $(P)$ are presented in each figure. The solid lines represent the fitted linear regression trendline.

Abbreviations: BMP4, plasma bone morphogenetic protein-4; IL-I $\beta$, interleukine-I $\beta$; TNF- $\alpha$, tumor necrosis factor- $\alpha$; IL-I0, interleukine-I0.

BMP4 levels protects pulmonary arteries from inflammatory injuries. ${ }^{22}$ Furthermore, BMP4 secreted by cancer cells will exert an immuno-suppressive effect via favoring anti-inflammatory M2 type macrophage nearby. ${ }^{23,24}$ One possible explanation for the discrepancy of BMP4's effect on inflammation is due to the dynamic changes of BMPRII (one type of BMP4 receptors) expressions. Recent studies have revealed that pro-inflammatory cytokines could downregulate BMPRII expression in endothelium during the pathogenesis of atherosclerosis. ${ }^{13,25,26}$ Moreover, the knockdown of BMPRII was shown to switch BMP4's effect from anti-inflammation to pro-inflammation. Above all, one can suggest that BMP4 plays a crucial role in the regulation of inflammation.

Although substantial evidence from cell and animal studies has revealed BMP4's regulation on inflammation, scant data is available regarding the circulating BMP4 levels in humans. One research reported that patients'
BMP4 serum levels were elevated after myocardial infarction. ${ }^{27}$ The BMP4 elevation lasted for about 14 days and was correlated with left ventricular remodeling. ${ }^{27}$ Another study furtherly proved that BMP4 plasma levels in patients suffering from out-of-hospital cardiac arrest were significantly higher than patients with stable coronary artery disease or healthy volunteers, accompanied by increased mortality and unfavorable neurological outcomes. ${ }^{28}$ Remarkably, the serum from patients undergoing myocardial infarction and postresuscitation was found to induce endothelial dysfunction via activating the BMP4 signaling pathway and to exert pro-inflammatory properties in vitro. ${ }^{11}$ All the evidence above suggests that circulating BMP4 in humans may play a key role in the process of tissue injury and repair.

To the best of our knowledge, the perioperative relationship between circulating BMP4 and inflammation has not been elucidated. In the present study, we have tested 
Table 2 Plasma Concentrations of BMP4, Noggin, and Inflammatory Cytokines Before Surgery (TI), at the End of the Surgery (T2), and 24 Hours After Surgery (T3)

\begin{tabular}{|c|c|c|c|c|}
\hline Control & TI & T2 & T3 & $P$-value \\
\hline BMP4, pg/mL & $4 \mid 4$ (359-447) & $75 \mid$ (705-819) & $532(472-643)$ & $<0.01$ \\
\hline $\mathrm{IL}-\mathrm{I} \beta, \mathrm{pg} / \mathrm{mL}$ & $142(125-175)$ & $317(266-427)$ & $211(193-249)$ & $<0.01$ \\
\hline TNF- $\alpha, p g / m L$ & $194(|5|-2 \mid 2)$ & $337(267-396)$ & $262(219-305)$ & $<0.01$ \\
\hline IL-10, pg/mL & $17 \mid(156-195)$ & $86(8|-| 17)$ & I38 (I28-|44) & $<0.01$ \\
\hline Noggin, pg/mL & $1542(1254-1916)$ & $1398(|197-| 721)$ & $1333(|| 82-1592)$ & 0.40 \\
\hline Flurb & TI & T2 & T3 & $P$-value \\
\hline BMP4, pg/mL & $510(462-543)$ & 710 (649-833) & $506(46 I-558)$ & $<0.01$ \\
\hline IL-I $\beta, p g / m L$ & $196(165-220)$ & $319(232-373)$ & $220(202-242)$ & $<0.01$ \\
\hline TNF- $\alpha, p g / m L$ & $185(167-240)$ & $358(257-422)$ & $238(219-296)$ & $<0.01$ \\
\hline IL-10, pg/mL & $182(164-200)$ & $97(8 \mathrm{I}-105)$ & $155(131-165)$ & $<0.01$ \\
\hline Noggin, pg/mL & 1749 (I I0I-2003) & 1482 (1217-1889) & 1408 (|169-1883) & 0.87 \\
\hline
\end{tabular}

Notes: Data presented as median (interquartile range). $P$-values calculated based on the Kruskal-Wallis test.

Abbreviations: BMP4, plasma bone morphogenetic protein-4; IL-I $\beta$, interleukine-I $\beta$; TNF- $\alpha$, tumor necrosis factor- $\alpha$; IL-I0, interleukine-I0; Flurb, flurbiprofen.

the plasma concentrations of BMP4 in each patient, finding that the baseline of patients suffering from lung cancer was mainly at $450-550 \mathrm{pg} / \mathrm{mL}$, which was significantly higher than the reference values of circulating BMP4 (100-200pg/mL) reported in healthy controls by previous researches. ${ }^{27-29}$ The possible reason can be attributed to the cancer secretion and systemic inflammatory status of the body. Moreover, we also detected the elevation of pro-inflammatory cytokines and the decline of anti-inflammatory cytokines both at the end of the surgery and 24 hours later, reflecting the initiation and long-lasting effect of inflammation caused by perioperative noxious stimuli. Simultaneously, we observed that plasma BMP4 levels were increased and maintained after surgery at a higher level, being strongly positively correlated with pro-inflammatory cytokines, including IL-1 $\beta$ and TNF- $\alpha$, but negatively correlated with anti-inflammatory factor IL-10. All the results above indicate that BMP4 might serve as a sensitive proinflammatory indicator.

COX-2 is another known inflammatory regulator and could be inducible by pro-inflammatory cytokines. ${ }^{30}$ Previous studies have reported that BMP4 could induce COX2 upregulation in endothelial cells in vitro, and

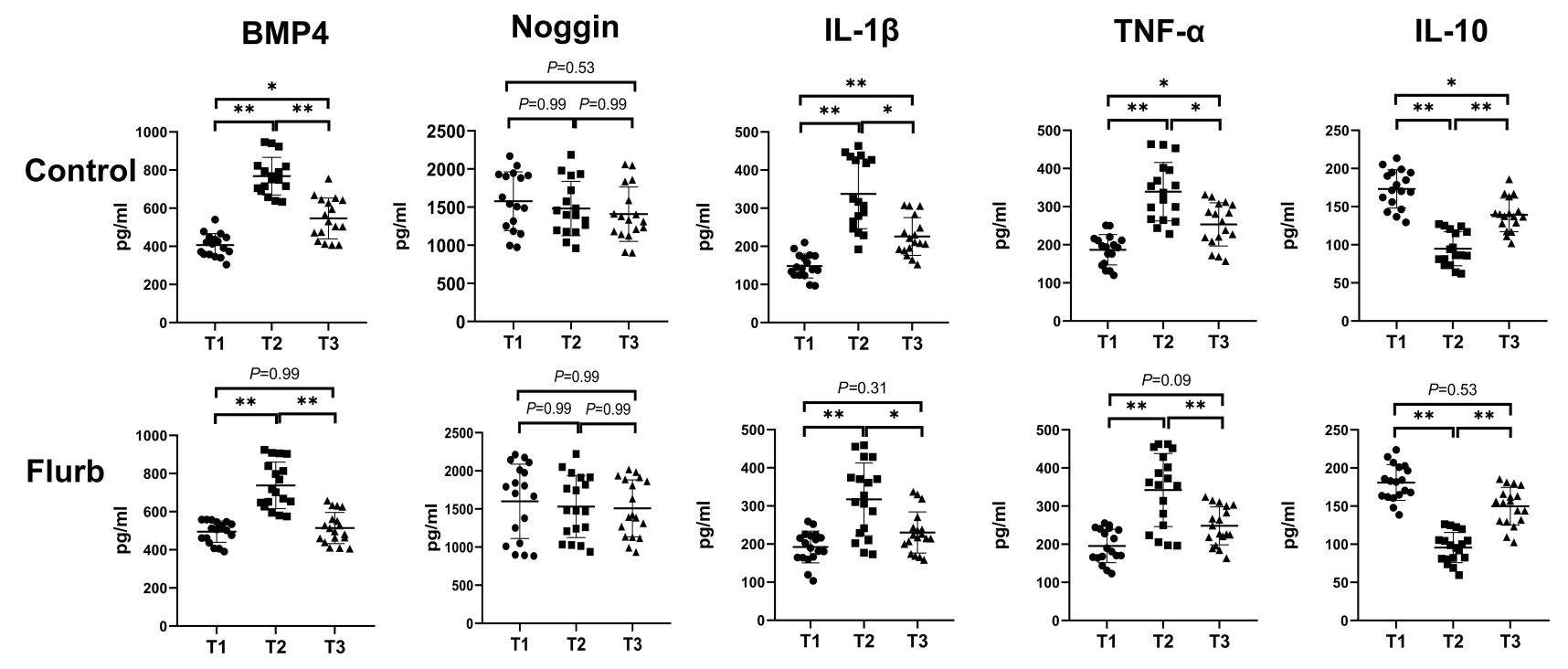

Figure 3 Boxplots for the plasma concentrations of BMP4, Noggin, and inflammatory cytokines before surgery (TI), at the end of the surgery (T2), and 24 hours after surgery (T3). The results of the Dunn's multiple comparison test are presented in each figure. *Indicate a $P$-value $<0.05$; **Indicate a $P$-value $<0.0$ I.

Abbreviations: BMP4, plasma bone morphogenetic protein-4; IL-I $\beta$, interleukine-I $\beta$; TNF- $\alpha$, tumor necrosis factor- $\alpha$; IL-I0, interleukine-I0; Flurb, flurbiprofen. 
Table 3 The Relative Changes of Plasma Concentrations of BMP4, Noggin, and Inflammatory Cytokines at the End of the Surgery (T2) and 24 Hours After Surgery (T3)

\begin{tabular}{|c|c|c|c|c|}
\hline & $\begin{array}{l}\text { Time } \\
\text { Point }\end{array}$ & $\begin{array}{l}\text { Control } \\
(N=\mid 7)\end{array}$ & $\begin{array}{c}\text { Flurb } \\
(\mathbf{N}=\mid 8)\end{array}$ & $P$-value \\
\hline \multirow[t]{2}{*}{ BMP4 } & $\mathrm{T} 2$ & $1.9(1.7-2.1)$ & $1.4(1.3-1.7)$ & 0.002 \\
\hline & T3 & $1.3(1.1-1.6)$ & $1.0(0.9-1.1)$ & 0.002 \\
\hline \multirow[t]{2}{*}{ IL-I $\beta$} & T2 & $2.4(1.9-2.8)$ & $1.8(1.4-2.0)$ & 0.006 \\
\hline & T3 & $1.6(1.2-1.8)$ & I.I (I.0-I.3) & 0.023 \\
\hline \multirow[t]{2}{*}{ TNF- $\alpha$} & $\mathrm{T} 2$ & $1.8(1.5-2.2)$ & $1.6(1.4-1.9)$ & 0.509 \\
\hline & T3 & $1.3(1.1-1.6)$ & $1.2(1.0-1.5)$ & 0.621 \\
\hline \multirow[t]{2}{*}{ IL-10 } & $\mathrm{T} 2$ & $0.6(0.4-0.7)$ & $0.5(0.4-0.6)$ & 0.766 \\
\hline & T3 & $0.8(0.7-0.9)$ & $0.8(0.7-0.9)$ & 0.597 \\
\hline \multirow[t]{2}{*}{ Noggin } & $\mathrm{T} 2$ & I.I (0.8-I.2) & I.I (0.8-I.2) & 0.644 \\
\hline & T3 & $0.9(0.7-1.1)$ & $1.0(0.6-1.4)$ & 0.597 \\
\hline
\end{tabular}

Notes: Data presented as median (interquartile range). $P$-values calculated based on the Mann-Whitney $U$-test. The relative changes indicated the ratio of the plasma concentrations at different time points versus the concentration before surgery (TI). Abbreviations: BMP4, plasma bone morphogenetic protein-4; IL-I $\beta$, interleukineI $\beta$; TNF- $\alpha$, tumor necrosis factor- $\alpha$; IL-10, interleukine-I0; Flurb, flurbiprofen.

hypertensive human renal arteries show higher levels of COX-2 and BMP4, accompanied by endothelial dysfunction. ${ }^{19,31}$ Similarly, we also found that the elevation of BMP4 and pro-inflammatory factors 24 hours after surgery could be alleviated by the application of flurbiprofen, a non-steroidal anti-inflammatory drug. What's more, flurbiprofen treatment decreased the relative changes of BMP4 and IL-1 $\beta$ from $\mathrm{T} 2$ to $\mathrm{T} 1$ and from $\mathrm{T} 3$ to $\mathrm{T} 1$. Thus, it prompted that circulating BMP4 might regulate inflammation clinically via COX-2 pathways.

Another key regulator of BMP4 signaling is Noggin, which directly binds with and de-activates BMP4 with high affinity. ${ }^{15}$ Strong evidence has already proven that Noggin application could exert anti-inflammatory properties by inhibiting NOX-1 upregulation and monocyte adhesion in the pathological process of BMP4-induced endothelial dysfunction ${ }^{19,32,33}$ and cardiomyocyte hypertrophy. ${ }^{34}$ In the present study, however, we found that endogenous Noggin levels stayed almost unchanged during surgery. The mechanism for such an approach remains unclear and whether Noggin can be a new therapeutic approach to treating inflammation needs further investigation.

Our study has several limitations. Firstly, we have only selected patients undergoing lobectomy, which may not be well representative of the whole general population. Secondly, we have calculated the planned sample size based on the correlations between BMP4 and other variables instead of the differences between Control and Flurb groups. Therefore, although we got some insignificant results regarding the relative changes of Noggin and other inflammatory cytokines between Control and Flurb groups, this might be due to inadequate sample size. Thirdly, we have not extended our investigation to define the effect of circulating BMP4 elevation on pulmonary arteries or tumors, considering that BMP4 would exert a diverse regulatory effect on inflammation in different organs. Future studies are required to determine the differential expressions of BMPRII at the lesion site, which might be important for determining the effects of BMP4.

Overall, the present study shows that circulating BMP4 is markedly elevated during surgery and highly correlated with inflammation cytokines. Moreover, the elevation of BMP4 and inflammatory cytokines could be alleviated by the applications of non-steroidal anti-inflammatory drugs, indicating that BMP4 may exert pro-inflammatory properties via the COX-2 signaling pathways.

\section{Data Sharing Statement}

All individual deidentified participant data and statistical analysis code that underlie the results reported in this article will be available after publication. Data can be requested for academic use by contacting the corresponding author (Lin Yang: linyang@csu.edu.cn).

\section{Author Contributions}

All authors contributed to data analysis, drafting or revising the article, gave final approval of the version to be published, agreed to the submitted journal, and agree to be accountable for all aspects of the work.

\section{Disclosure}

The authors report no conflicts of interest in this work.

\section{References}

1. Rossaint J, Zarbock A. Perioperative inflammation and its modulation by anesthetics. Anesth Analg. 2018;126(3):1058-1067. doi:10.1213/ ANE.0000000000002484

2. Frangogiannis NG. The inflammatory response in myocardial injury, repair, and remodelling. Nat Rev Cardiol. 2014;11(5):255-265. doi: $10.1038 /$ nrcardio. 2014.28

3. Hilfiker-Kleiner D, Shukla P, Klein G, et al. Continuous glycoprotein-130-mediated signal transducer and activator of transcription-3 activation promotes inflammation, left ventricular rupture, and adverse outcome in subacute myocardial infarction. Circulation. 2010;122(2):145-155. doi:10.1161/CIRCULATI ONAHA.109.933127 
4. Boehm O, Baumgarten G, Hoeft A. Epidemiology of the high-risk population: perioperative risk and mortality after surgery. Curr Opin Crit Care. 2015;21(4):322-327. doi:10.1097/MCC.00000000 00000221

5. Cai J, Pardali E, Sánchez-Duffhues G, Ten Dijke P. BMP signaling in vascular diseases. FEBS Lett. 2012;586(14):1993-2002. doi:10.1016/ j.febslet.2012.04.030

6. Cole AE, Murray SS, Xiao J. Bone morphogenetic protein 4 signalling in neural stem and progenitor cells during development and after injury. Stem Cells Int. 2016;2016.

7. de Vinuesa AG, Abdelilah-Seyfried S, Knaus P, Zwijsen A, Bailly S. BMP signaling in vascular biology and dysfunction. Cytokine Growth Factor Rev. 2016;27:65-79. doi:10.1016/j.cytogfr.2015.12.005

8. Yin W, Jo H, Voit EO. Systems analysis of the role of bone morphogenic protein 4 in endothelial inflammation. Ann Biomed Eng. 2010;38(2):291-307. doi:10.1007/s10439-009-9822-y

9. Noseda M, Peterkin T, Simões FC, Patient R, Schneider MD. Cardiopoietic factors: extracellular signals for cardiac lineage commitment. Circ Res. 2011;108(1):129-152. doi:10.1161/ CIRCRESAHA.110.223792

10. Qian S-W, Wu M-Y, Wang Y-N, et al. BMP4 facilitates beige fat biogenesis via regulating adipose tissue macrophages. $\mathrm{J} \mathrm{Mol} \mathrm{Cell}$ Biol. 2019;11(1):14-25. doi:10.1093/jmcb/mjy011

11. Helbing T, Arnold L, Wiltgen G, et al. Endothelial BMP4 regulates leukocyte diapedesis and promotes inflammation. Inflammation. 2017;40(6):1862-1874. doi:10.1007/s10753-017-0627-0

12. Hanna A, Frangogiannis NG. The role of the TGF- $\beta$ superfamily in myocardial infarction. Front Cardiovasc Med. 2019;6:140. doi:10.3389/fcrm.2019.00140

13. Wang D, Prakash J, Nguyen P, et al. Bone morphogenetic protein signaling in vascular disease: anti-inflammatory action through myocardin-related transcription factor A. J Biol Chem. 2012;287 (33):28067-28077. doi:10.1074/jbc.M112.379487

14. Jo H, Song H, Mowbray A. Role of NADPH oxidases in disturbed flow-and BMP4-induced inflammation and atherosclerosis. Antioxid Redox Signal. 2006;8(9-10):1609-1619. doi:10.1089/ars.20 06.8.1609

15. Zimmerman LB, De Jesús-escobar JM, Harland RM. The Spemann organizer signal noggin binds and inactivates bone morphogenetic protein 4. Cell. 1996;86(4):599-606. doi:10.1016/S0092-8674(00) 80133-6

16. Kloen P, Lauzier D, Hamdy RC. Co-expression of BMPs and BMP-inhibitors in human fractures and non-unions. Bone. 2012;51 (1):59-68. doi:10.1016/j.bone.2012.03.032

17. Rajski M, Saaf A, Buess M. BMP2 response pattern in human lung fibroblasts predicts outcome in lung adenocarcinomas. BMC Med Genomics. 2015;8(1):1-12. doi:10.1186/s12920-015-0090-4

18. Chattopadhyay T, Singh RR, Gupta S, Surolia A. Bone morphogenetic protein-7 (BMP-7) augments insulin sensitivity in mice with type II diabetes mellitus by potentiating PI3K/AKT pathway. Biofactors. 2017;43(2):195-209. doi:10.1002/biof.1334

19. Youn J-Y, Zhou J, Cai H. Bone morphogenic protein 4 mediates NOX1-dependent eNOS uncoupling, endothelial dysfunction, and COX2 induction in type 2 diabetes mellitus. Mol Endocrinol. 2015;29(8):1123-1133. doi:10.1210/ME.2014-1313

20. Wu T, Ling Q-Y, Zhong C, et al. Expression of BMP4 in myocardium and vascular tissue of obese mice. J Inflamm. 2015;12(1):1-6. doi:10.1186/s12950-015-0047-6
21. Mukaka MM. A guide to appropriate use of correlation coefficient in medical research. Malawi Med J. 2012;24(3):69-71.

22. Csiszar A, Labinskyy N, Jo H, Ballabh P, Ungvari Z. Differential proinflammatory and prooxidant effects of bone morphogenetic protein-4 in coronary and pulmonary arterial endothelial cells. $\mathrm{Am}$ J Physiol Heart Circ Physiol. 2008;295(2):H569-H577. doi:10.1152/ ajpheart.00180.2008

23. Martínez VG, Rubio C, Martínez-Fernández M, et al. BMP4 induces M2 macrophage polarization and favors tumor progression in bladder cancer. Clin Cancer Res. 2017;23(23):7388-7399. doi:10.1158/10780432.CCR-17-1004

24. Valencia J, Fernández-Sevilla L, Fraile-Ramos A, et al. Acute lymphoblastic leukaemia cells impair dendritic cell and macrophage differentiation: role of BMP4. Cells. 2019;8(7):722. doi:10.3390/ cells 8070722

25. Kariyawasam HH, Xanthou G, Barkans J, Aizen M, Kay AB, Robinson DS. Basal expression of bone morphogenetic protein receptor is reduced in mild asthma. Am J Respir Crit Care Med. 2008;177 (10):1074-1081. doi:10.1164/rccm.200709-1376OC

26. Kim CW, Song H, Kumar S, et al. Anti-inflammatory and antiatherogenic role of BMP receptor II in endothelial cells. Arterioscler Thromb Vasc Biol. 2013;33(6):1350-1359. doi:10.1161/ ATVBAHA.112.300287

27. Kercheva M, Gusakova AM, Ryabova TR, Suslova TE, Kzhyshkowska J, Ryabov VV. Serum levels of bone morphogenetic proteins 2 and 4 in patients with acute myocardial infarction. Cells. 2020;9(10):2179. doi:10.3390/cells9102179

28. Arnold L, Weberbauer M, Herkel M, et al. Endothelial BMP4 promotes leukocyte rolling and adhesion and is elevated in patients after survived out-of-hospital cardiac arrest. Inflammation. 2020;43 (6):2379-2391. doi:10.1007/s10753-020-01307-9

29. Penn M, Mausner-Fainberg K, Golan M, Karni A. High serum levels of BMP-2 correlate with BMP-4 and BMP-5 levels and induce reduced neuronal phenotype in patients with relapsing-remitting multiple sclerosis. J Neuroimmunol. 2017;310:120-128. doi:10.1016/j. jneuroim.2017.07.008

30. Eligini S, Stella Barbieri S, Cavalca V, et al. Diversity and similarity in signaling events leading to rapid Cox-2 induction by tumor necrosis factor- $\alpha$ and phorbol ester in human endothelial cells. Cardiovasc Res. 2005;65(3):683-693. doi:10.1016/j.cardiores.2004.10.024

31. Wong WT, Tian XY, Chen Y, et al. Bone morphogenic Protein-4 impairs endothelial function through oxidative stress-dependent cyclooxygenase-2 upregulation: implications on hypertension. Circ Res. 2010;107(8):984-991. doi:10.1161/CIRCRESAHA.110.222794

32. Sorescu GP, Sykes M, Weiss D, et al. Bone morphogenic protein 4 produced in endothelial cells by oscillatory shear stress stimulates an inflammatory response. J Biol Chem. 2003;278(33):31128-31135. doi:10.1074/jbc.M300703200

33. Sorescu GP, Song H, Tressel SL, et al. Bone morphogenic protein 4 produced in endothelial cells by oscillatory shear stress induces monocyte adhesion by stimulating reactive oxygen species production from a nox1-based NADPH oxidase. Circ Res. 2004;95 (8):773-779. doi:10.1161/01.RES.0000145728.22878.45

34. Sun B, Sheng Y, Huo R, et al. Bone morphogenetic protein-4 contributes to the down-regulation of $\mathrm{Kv} 4.3 \mathrm{~K}+$ channels in pathological cardiac hypertrophy. Biochem Biophys Res Commun. 2013;436 (4):591-594. doi:10.1016/j.bbrc.2013.05.113 


\section{Publish your work in this journal}

The Journal of Inflammation Research is an international, peerreviewed open-access journal that welcomes laboratory and clinical findings on the molecular basis, cell biology and pharmacology of inflammation including original research, reviews, symposium reports, hypothesis formation and commentaries on: acute/chronic inflammation; mediators of inflammation; cellular processes; molecular mechanisms; pharmacology and novel anti-inflammatory drugs; clinical conditions involving inflammation. The manuscript management system is completely online and includes a very quick and fair peerreview system. Visit http://www.dovepress.com/testimonials.php to read real quotes from published authors. 